\title{
Comportamento ingestivo de novilhos Nelore em fases da terminação distintas, recebendo dietas de alto grão com ureia encapsulada ou não
}

Ingestive behavior of Nellore steers at different finishing stages, receiving high-grain diets with encapsulated urea or not

Comportamiento ingestivo de novillos Nellore en diferentes etapas de acabado, recibiendo dietas de alto grano con o sin urea encapsulada

Raul Dirceu Pazdiora

ORCID: https://orcid.org/0000-0002-5495-4737

Fundação Universidade Federal de Rondônia, Brasil

E-mail: raul.pazdiora@unir.br

Marcelo Henrique de Faria

ORCID: https://orcid.org/0000-0001-9369-2496

Agência Paulista de Tecnologia dos Agronegócios, Brasil

E-mail: mfaria@apta.sp.gov.br

Bruna Rafaela Caetano Nunes Pazdiora

ORCID: https://orcid.org/0000-0002-5262-0514

Fundação Universidade Federal de Rondônia, Brasil

E-mail: bruna.nunes@unir.br

José Keoma Salles Santos

ORCID: https://orcid.org/0000-0002-7588-597X

Fundação Universidade Federal de Rondônia, Brasil

E-mail: josekeoma82@gmail.com

Marcus Vinicius da Silva

ORCID: https://orcid.org/0000-0002-1501-6342

Fundação Universidade Federal de Rondônia, Brasil

E-mail: marcus.1998mvs@gmail.com

Vanessa Dib

ORCID: https://orcid.org/0000-0002-6224-2940 Zootecnista, Brasil

E-mail: vanessadibb@outlook.com

Felipe Oliveira do Carmo

ORCID: https://orcid.org/0000-0002-2791-1045 Zootecnista, Brasil

E-mail: felipeoliveiracarmo@hotmail.com

José Eduardo dos Santos Melo de Souza

ORCID: https://orcid.org/0000-0003-2946-2789 Empresa Work\&Trip Exchange Internacional, Ucrânia

E-mail: jesmelos@hotmail.com

Ricardo Rivas

ORCID: https://orcid.org/0000-0001-9281-0163 Zootecnista, Brasil

E-mail: rivas.zootec@gmail.com

Luiz Henrique Ferreira

ORCID: https://orcid.org/0000-0001-6191-5018 Zootecnista, Brasil

E-mail: luizhenriquef84@gmail.com

Antônio Rafael Camargo Rodrigues

ORCID: https://orcid.org/0000-0003-0273-2478 Zootecnista, Brasil

E-mail: rcamargo.barretos@live.com

João Moreira Castro

ORCID: https://orcid.org/ 0000-0002-5088-3652 Zootecnista, Brasil

E-mail: jonny_castro20@hotmail.com

Flávio Dutra de Resende

ORCID: https://orcid.org/0000-0001-6999-9981 


\section{Resumo}

Objetivou-se avaliar o comportamento ingestivo de novilhos Nelores alimentados com dietas contendo ureia encapsulada ou não, em quatro períodos da terminação dos animais confinados. Foram utilizados 32 e $28 ; 24$ e $21 ; 16$ e $14 ; 8$ e 7 animais para as dietas sem e com ureia encapsulada nos $1^{\circ} ; 2^{\circ} ; 3^{\circ}$ e $4^{\circ}$ períodos, respectivamente. Cada período foi composto por 50 dias, com exceção do último que teve 45 dias. Os animais foram confinados em baias individuais. Cada avaliação, dentro do período, foi de $48 \mathrm{~h}$ consecutivas, com intervalos de observação de 10 minutos. $\mathrm{O}$ delineamento experimental foi inteiramente casualizado em arranjo fatorial $2 \times 4$ e, para a comparação das médias, foi utilizado o teste "t" de student. Houve interação entre dietas e as avaliações nos períodos para o consumo e eficiência de ruminação da matéria seca, e para as demais variáveis estudadas não ocorreu interação. O tempo de ócio foi maior e o de ruminação foi menor para os animais que receberam a dieta sem ureia encapsulada em relação à dieta com ureia encapsulada. O tempo de alimentação dos animais foi maior para a avaliação do segundo período, porém não diferem do primeiro e quarto períodos. O tempo de ócio foi maior na avaliação do segundo período e semelhante com a avaliação no terceiro período. O comportamento ingestivo é alterado em diferentes períodos do confinamento e pela composição da dieta.
\end{abstract}

Palavras-chave: Bovinos; Confinamento; Eficiência de alimentação; Etologia; Ócio; Ruminação.

\begin{abstract}
The objective was to evaluate the ingestive behavior of Nellore steers fed with diets containing encapsulated urea or not, in four periods of the termination of the confined animals. 32 and 28 were used; 24 and 21; 16 and 14; 8 and 7 animals for diets without and with urea encapsulated in the 1st; 2nd; 3rd and 4th periods, respectively. Each period consisted of 50 days, with the exception of the last period that had 45 days. The animals were confined in individual pens. Each evaluation, within the period, was 48 consecutive hours, with 10-minute observation intervals. The experimental design was completely randomized in a $2 \times 4$ factorial arrangement and for the comparison of means, the student's " $t$ " test was used. There was interaction between diets and evaluations in the periods for dry matter consumption and rumination efficiency, and for the other variables studied there was no interaction. The leisure time was longer and the rumination time was shorter for the animals that received the diet without encapsulated urea in relation to the diet with encapsulated urea. The feeding time of the animals was longer for the evaluation of the second period, but it did not differ from the first and fourth periods. Leisure time was longer in the second period and similar to the third period. Ingestive behavior is altered at different periods of confinement and by the composition of the diet.
\end{abstract} Keywords: Cattle; Feedlot; Feed efficiency; Ethology; Leisure; Rumination.

\title{
Resumen
}

El objetivo fue evaluar el comportamiento de novillos Nellore alimentados con dietas que contienen urea encapsulada o no, em cuatro períodos de la terminación de los animales confinados. Fueron utilizados 32 y $28 ; 24$ y $21 ; 16$ y $14 ; 8$ y 7 animales para las dietas sin y con urea encapsulada en $\operatorname{los} 1^{\circ} ; 2^{\circ} ; 3^{\circ}$ y $4^{\circ}$ períodos, respectivamente. Cada período fue cumpuesto por 50 días, excepto el último, que comprendió 45 días. Los animals fueran confinados en establos individuales. Cada evaluación, dentro del período fue de $48 \mathrm{~h}$ consecutivas, con intervalos de observación de 10 minutos. El deseño experimental fue interamento al azar, em arreglo factorial 2 x 4 y, para la comparación de las medias se utilize el test " $\mathrm{t}$ " de student. Hubo interacción entre dietas y las evaluaciones en los períodos para el consume y eficiencia de rumia da matéria seca, y para las demás variables estudiadas no hubo interacción. El tiempo de ocio fue más grande y el de rumia ha sido más pequeño para los animales que recebieron la dieta sin urea encapsulada, con relación a la dieta com urea encapsulada. El tiempo de alimentación de los animales he sido más grande para la evaluación del segundo período, sin embargo, no difieren del primeiro ni del cuarto período. El tiempo de ocio fue más grande en la evaluación del segundo período y similar com la evaluación en el tercer período. El comportamiento ingestivo es alterado en diferentes períodos del confinamiento y por la composición de la dieta.

Palabras clave: Bovinos; Confinamiento; Eficiencia de la alimentación; Etologia; Ocio; Rumia.

\section{Introdução}

A ingestão de dietas ricas em fibras acompanhou todo o processo evolutivo dos animais ruminantes, uma vez que forragens, especialmente gramíneas, compõem grande parte da dieta desses animais (Paulino et al., 2010). No entanto, nas últimas décadas iniciou-se um processo de intensificação da pecuária, com melhorias na gestão da atividade e novos enfoques em genética, sanidade e nutrição. E neste contexto, a evolução das dietas ofertadas para os ruminantes, com destaque para os bovinos, sofreu mudanças bruscas, já que em muitas situações os animais são alimentados sem o uso de volumoso, principalmente durante a fase de terminação. Esta evolução pode ser observada em levantamento realizado por Pinto e Millen (2016), em que entrevistaram 33 nutricionistas que respondiam por aproximadamente 95\% dos bovinos confinados no Brasil, verificaram média de uso de volumoso de $206 \mathrm{~g} \mathrm{~kg}^{-1}$ de matéria seca na dieta, semelhante ao relatado por Oliveira e Millen 
(2014), mas inferior às $288 \mathrm{~g} \mathrm{~kg}^{-1}$ de matéria seca relatadas por Millen et al. (2009). Em relação ao nível de inclusão do concentrado na dieta, 90,9\% dos participantes recomendaram de utilizam de 710 a $900 \mathrm{~g} \mathrm{~kg}^{-1}$ de matéria seca da dieta.

Independente do sistema de terminação, em confinamento ou a pasto, o uso de dietas de alto grão é uma prática crescente em sistemas de produção de carne bovina. Segundo Paulino et al. (2010), com a evolução das unidades de engorda intensiva no Brasil, que passaram a atingirem maiores escalas, a dependência de alimentos volumosos passa a ser um entrave. Além disso, segundo Santos et al. (2011), a substituição de alimentos que contenham proteína verdadeira por alimentos com maior teor de nitrogênio na forma de nitrogênio não proteico, como a ureia, pode, sem alterar o desempenho dos animais, melhorar a eficiência financeira da alimentação, reduzindo a necessidade de compra de concentrados proteicos.

Porém a ágil solubilização da ureia resulta em perdas de nitrogênio, reduzindo a capacidade de síntese microbiana ruminal o que pode levar à riscos de intoxicação se o potencial de absorção de nitrogênio for maior que a capacidade de síntese dos microorganismos. Nesse sentido, a utilização de ureia encapsulada, que possui uma fina camada de proteção, proporciona uma liberação mais lenta, podendo resultar numa absorção do nitrogênio mais constante e adequada, melhorando o desempenho dos animais (Ferreira et al., 2005).

Com toda esta evolução, o entendimento do comportamento ingestivo é de grande importância para estudos com bovinos mantidos em regime de confinamento. Callegaro et al. (2018) descrevem que o confinamento e suas alternativas alimentares compreendem na atualidade o principal meio de estudo da etologia, não devido apenas à importância e ao crescimento verificado nos últimos anos, mas pela possibilidade de dissecação do comportamento animal, realizada através de avaliações mais complexas. E dessa forma, quando surge uma nova proposta alimentar, é necessário investigar não apenas a resposta do desempenho animal, mas o respectivo efeito no comportamento ingestivo diário.

Segundo Mertens (1994), o consumo de alimento pode ser influenciado por fatores associados aos alimentos, como palatabilidade, textura, aparência visual, bem como aos fatores e às condições interligadas aos animais, tais como estado emocional, interações e aprendizado. Avaliar estes aspectos através do comportamento pode explicar variações no consumo de alimento e desempenho produtivo, e com isto, proporcionar alternativas visando melhorar a eficiência. Neste sentido, objetivou-se avaliar o comportamento ingestivo de novilhos Nelores alimentados com dietas contendo ureia encapsulada ou não em quatro fases ao longo da terminação dos animais confinados.

\section{Metodologia}

Os animais foram confinados no Pólo Regional de Desenvolvimento Tecnológico dos Agronegócios da Alta Mogiana, situado no município de Colina, estado de São Paulo, Brasil, pertencentes à Agência Paulista de Tecnologia dos Agronegócios, no período de março a outubro.

Foram testadas duas dietas com ureia: encapsulada ou não, em diferentes fases de alimentação, que foram divididas em períodos: primeiro período que consistia nos 50 dias iniciais, o segundo período, dos 51 até 100 dias de alimentação, o terceiro período, dos 101 aos 150 dias de alimentação e quarto período dos 151 aos 195 dias de alimentação. A ureia encapsulada foi da marca comercial Optigen ${ }^{\circledR}$ (Alltech). O número de animais utilizados foi diferente por tratamento em função da escolha inicial dos lotes para serem abatidos, 15 por tratamento, nos diferentes pesos corporais $455 ; 485 ; 555$ e $580 \mathrm{~kg}$. Assim, foram utilizados 32 e $28 ; 24$ e $21 ; 16$ e 14 e 8 e 7 animais para as dietas sem e com ureia encapsulada no $1^{\circ} ; 2^{\circ} ; 3^{\circ}$ e $4^{\circ}$ períodos de alimentação, respectivamente. Os animais foram confinados em baias individuais de $10 \mathrm{~m}^{2}$ parcialmente cobertos, com piso de alvenaria, bebedouro regulado por torneira-bóia e disponibilidade de $2 \mathrm{~m}$ linear de cocho por animal.

Na Figura 1 são apresentadas as condições meteorológicas durante o período experimental. 
Figura 1. Dados climáticos no período experimental.

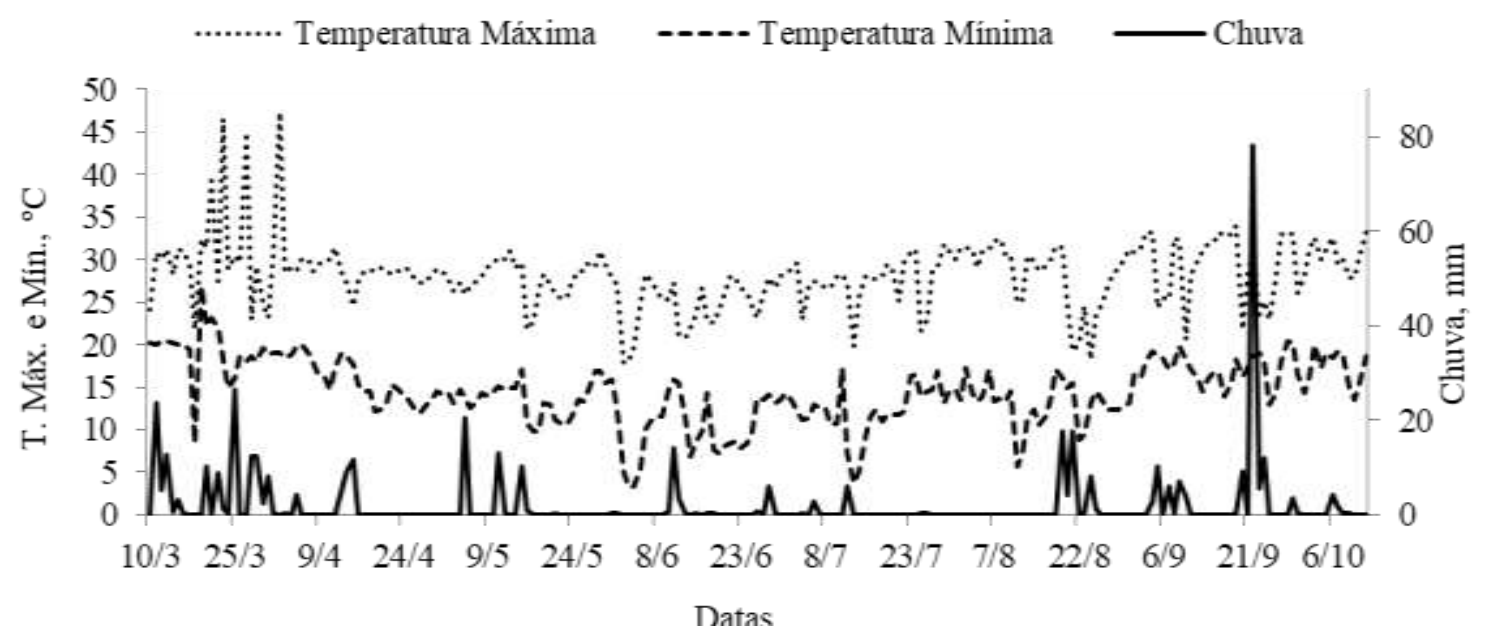

Fonte: CIIAGRO - Centro integrado de informações agrometeorológicas.

As dietas foram formuladas com o objetivo inicial de atender à exigência para o ganho de peso médio diário de 1,500 kg de acordo com o software para a formulação Ração de Lucro Máximo (RLM, versão 3.0), conforme a Tabela 1 . O grão de milho foi triturado em peneira com malha de $6 \mathrm{~mm}$. O fornecimento do alimento foi realizado duas vezes ao dia, sendo $50 \%$ no período da manhã ( 8 horas) e o restante, na parte da tarde (14 horas). A quantidade de alimento fornecida por dia foi regulada procurando manter as sobras em $10 \%$ da quantidade ofertada.

Tabela 1. Composição da dieta utilizada para a alimentação dos animais experimentais.

\begin{tabular}{lccccccc}
\hline & \multicolumn{7}{c}{ Composição alimentar, g/kg de matéria seca } \\
\cline { 2 - 8 } Dietas & $\begin{array}{c}\text { Bagaço de } \\
\text { cana cru }\end{array}$ & $\begin{array}{c}\text { Grão de } \\
\text { milho }\end{array}$ & $\begin{array}{c}\text { Caroço de } \\
\text { algodão }\end{array}$ & $\begin{array}{c}\text { Farelo de } \\
\text { soja }\end{array}$ & Ureia & $\begin{array}{c}\text { Ureia } \\
\text { encapsulada }\end{array}$ & $\begin{array}{c}\text { Núcleo } \\
\text { Mineral* }^{*}\end{array}$ \\
\hline SUE & 130,0 & 674,2 & 99,4 & 50,0 & 12,5 & 0,00 & 33,9 \\
CUE & 200,0 & 584,2 & 99,4 & 70,0 & 2,50 & 10,0 & 33,9 \\
\hline \multirow{2}{*}{ Dietas } & MS & MM & PB & EE & FDN & FDA & NDT \\
\hline SUE & 814,7 & 75,4 & 140,7 & 55,4 & 242,6 & 143,4 & 682,4 \\
CUE & 802,5 & 83,2 & 141,7 & 54,9 & 302,4 & 187,2 & 687,8 \\
\hline
\end{tabular}

SUE $=$ Dieta sem ureia encapsulada $; \mathrm{CUE}=$ Dieta com ureia encapsulada;

$\mathrm{MS}=$ matéria seca; $\mathrm{MM}=$ matéria mineral; $\mathrm{PB}=$ =proteína bruta; $\mathrm{EE}=$ extrato etéreo; FDN=fibra detergente neutro; FDA=fibra detergente ácido; NDT=nutrientes digestíveis totais;

*Composição: vitamina A = 78000 UI; Vitamina $D_{3}=9750$ UI; Vitamina E = 325 UI; Cálcio = 160 g; Fósforo = 18,75 g; Sódio = 62,5 g; Potássio = $50 \mathrm{~g}$; Magnésio $=15 \mathrm{~g} ;$ Enxofre $=25 \mathrm{~g}$; Manganês $=780 \mathrm{mg}$; Zinco = $1875 \mathrm{mg}$; Cobre $=390 \mathrm{mg}$; Cobalto $=23,6 \mathrm{mg}$; Iodo $=23,6 \mathrm{mg}$; Selênio $=6,5 \mathrm{mg}$; Monensina sódica $=780 \mathrm{mg}$;

Fonte: Autores.

A adaptação dos animais à dieta foi realizada de forma restrita, em que a quantidade de alimento foi aumentada gradativamente, iniciando com $1 \%$ do peso corporal nos três primeiros dias e aumentando $0,2 \%$ a cada três dias, até o vigésimo primeiro dia. Posteriormente, o fornecimento foi à vontade até o vigésimo oitavo dia. Em seguida, iniciou-se o período de avaliação, que teve dias diferentes conforme os pesos de abate pretendidos. O consumo de matéria seca por animal foi 
determinado pela quantidade de alimento fornecido menos a sobra, avaliados durante os períodos de observação do comportamento ingestivo.

$\mathrm{O}$ registro de comportamento teve quatro avaliações de $48 \mathrm{~h}$ consecutivas em cada período. As 48 horas foram realizadas no último final de semana de cada período. As observações comportamentais foram realizadas de forma direta, com registro instantâneo e amostragem focal e cronometrado. Adotou-se o intervalo entre amostragens de 10 em 10 minutos, por dois observadores independentes em sistema de revezamento, posicionados estrategicamente de forma a não incomodar os animais. O turno de avaliação para cada dupla de observadores foi de 6 horas. As anotações foram feitas individualmente para cada animal, em que foram observados os seguintes parâmetros: tempo de alimentação, ócio, ruminação e ingestão de água. A eficiência de ingestão da matéria seca foi determinada pela razão entre o consumo de matéria seca e o tempo de alimentação e a eficiência de ruminação da matéria seca foi determinada pela razão do consumo de matéria seca e o tempo de ruminação.

Durante a coleta de dados, na observação noturna dos animais, o ambiente foi mantido com iluminação artificial. Os dados foram registrados em planilhas próprias.

As amostras dos ingredientes da dieta e as sobras foram coletadas todos os dias das avaliações de comportamento ingestivo. Estas foram pré-secadas em estufa de circulação forçada de ar a $55^{\circ} \mathrm{C}$ por 72 horas para a determinação da matéria parcialmente seca. Em seguida, foram moídas em moinho tipo Willey, com peneira com crivos de $1 \mathrm{~mm}$ e armazenadas para posterior análise. A matéria seca foi determinada em estufa a $105^{\circ} \mathrm{C}$ durante 16 horas (Silva \& Queiroz, 2002).

$\mathrm{O}$ delineamento experimental utilizado foi o inteiramente casualizado em arranjo fatorial $4 \times 2$ (quatro períodos de confinamento e duas dietas). Os dados foram submetidos ao teste de normalidade, pelo teste Shaphiro-Wilk. Os dados foram submetidos à análise de variância e para a comparação das médias foi utilizado o teste " $\mathrm{t}$ " de student, através do programa estatístico SAS (2001), em que se adotou $\alpha=0,05$.

\section{Resultados e Discussão}

Para os tempos de alimentação, ócio, ruminação e ingestão de água não houveram interações entre as dietas e as avaliações nos períodos de alimentação, em função disso, os dados foram analisados separadamente.

O consumo de matéria seca apresentou interação entre as avaliações dos períodos e as dietas, em que foram observados maior consumo de matéria seca no primeiro período para os animais que receberam a dieta sem ureia encapsulada em relação a dieta com ureia encapsulada. Nas avaliações do segundo e terceiro períodos verificaram maiores consumos para os animais que receberam a dieta com ureia encapsulada em relação a dieta sem ureia encapsulada, e na avaliação do quarto período o consumo foi semelhante entre as dietas. $\mathrm{Na}$ avaliação do segundo período, o consumo foi superior as demais avaliações independentes das dietas. Os dados de literatura apresentam variações de consumo com animais permanecendo mais tempo em confinamento (Jorge et al., 1997; Mello et al., 2010), assim como semelhança de consumo (Arboitte et al., 2004; Cruz et al. 2004). Pode-se citar como fatores que influenciam nestas variações de resultados, os grupos genéticos avaliados, tipos e tempo de alimentação, entre outros fatores. 
Tabela 2. Consumo de matéria seca no período de avaliação, kg/dia, de bovinos da raça Nelore não castrados alimentados com ureia, encapsulada (CUE) ou não (SUE), conforme os períodos de avaliação em confinamento.

\begin{tabular}{|c|c|c|c|c|c|c|c|c|c|}
\hline \multirow{2}{*}{ Dieta (D) } & \multicolumn{4}{|c|}{ Períodos $(\mathrm{P})$} & \multirow[b]{2}{*}{ Médias } & \multirow{2}{*}{$\mathrm{CV}(\%)$} & \multicolumn{3}{|c|}{ Probabilidade } \\
\hline & $1^{\circ}$ & $2^{\circ}$ & $3^{\circ}$ & $4^{\circ}$ & & & $\mathrm{P}$ & $\mathrm{D}$ & $\mathrm{P} * \mathrm{D}$ \\
\hline SUE & $9,13^{c}$ & $9,68^{b}$ & $8,00^{\mathrm{de}}$ & $7,69^{\mathrm{e}}$ & 8,62 & \multirow{2}{*}{12,3} & \multirow{2}{*}{$<0,0001$} & \multirow{2}{*}{0,3014} & \multirow{2}{*}{$<0,0001$} \\
\hline CUE & $8,37^{\mathrm{d}}$ & $10,17^{\mathrm{a}}$ & $8,93^{c}$ & $7,64^{\mathrm{e}}$ & 8,78 & & & & \\
\hline Médias & 8,75 & 9,93 & 8,47 & 7,66 & & & & & \\
\hline
\end{tabular}

a,b,c,d,e Médias com letras distintas na linha, diferem pelo teste $t$ de student ao nível de 5\%.

$\mathrm{CV}=$ coeficiente de variação;

Fonte: Autores.

O tempo de alimentação foi menor na avaliação do terceiro período, sendo maior e semelhante entre as demais avaliações (Tabela 2). Guimarães et al. (2020), em seu estudo de meta-análise, verificaram que o tempo médio gasto pelos animais em alimentação no sistema confinado foi de 249,39 min/dia (4,15 horas/dia), valor esse que possui amplitude de acordo com o tipo de dieta fornecida. Segundo Arnold (1985), os ruminantes procuram ajustar o consumo alimentar às suas necessidades nutricionais, principalmente de energia. No entanto, esta afirmação não foi observada no presente estudo, visto que, com o aumento do peso dos animais em confinamento e consequentemente, do número de dias confinados, ocorrem alterações nas necessidades nutricionais de mantença e produção (NRC, 2016), refletindo no ganho de peso. Apesar de adotar o mesmo protocolo durante a condução das atividades, pequenas alterações pontuais podem estar influenciando nesta variação de consumo ao longo dos dias de confinamento como as condições ambientais.

Um fator importante relacionado ao consumo de alimento é o hormônio leptina, que pode explicar a redução de consumo conforme aumentaram os dias que os animais ficaram confinados e consequentemente a deposição de gordura. Segundo Silva (2011), a leptina é produzida nas células adiposas e atua como regulador da ingestão de alimentos bem como nas reservas corporais, e sua síntese é regulada pelos principais hormônios que controlam a atividade do tecido adiposo. Além do efeito sobre a redução da ingestão de alimento, a leptina tem um forte efeito sobre o metabolismo do corpo, ela também aumenta a liberação do hormônio estimulante da tireoide, resultando na produção de tireoxina pela glândula tiroide (Silva, 2011). Delavaid et al. (2000) observaram correlação positiva entre gordura corporal subcutânea e leptina circulante em animais domésticos.

Tabela 3. Comportamento ingestivo de bovinos da raça Nelore não castrados alimentados com ureia, encapsulada (CUE) ou não (SUE), conforme os períodos de alimentação em confinamento

\begin{tabular}{|c|c|c|c|c|c|c|c|c|c|c|}
\hline \multirow{2}{*}{ Variáveis } & \multicolumn{4}{|c|}{ Períodos $(\mathrm{P})$} & \multicolumn{2}{|c|}{ Dieta (D) } & \multirow{2}{*}{$\begin{array}{l}\mathrm{CV} \\
(\%)\end{array}$} & \multicolumn{3}{|c|}{ Probabilidade } \\
\hline & $1^{\circ}$ & $2^{\circ}$ & $3^{\circ}$ & $4^{\circ}$ & CUE & SUE & & $\mathrm{P}$ & $\mathrm{D}$ & $\mathrm{P} * \mathrm{D}$ \\
\hline TA, h & $3,55^{\mathrm{a}}$ & $3,67^{\mathrm{a}}$ & $3,13^{\mathrm{b}}$ & $3,50^{\mathrm{a}}$ & 3,50 & 3,42 & 22,5 & 0,0006 & 0,4482 & 0,9343 \\
\hline $\mathrm{TR}, \mathrm{h}$ & $5,35^{\mathrm{a}}$ & $4,67^{\mathrm{b}}$ & $5,44^{\mathrm{a}}$ & $5,33^{\mathrm{a}}$ & 5,40 & 5,00 & 20,0 & $<0,0001$ & 0,0040 & 0,2306 \\
\hline TO, h & $14,8^{c}$ & $15,4^{\mathrm{a}}$ & $15,2^{\mathrm{ab}}$ & $14,9^{\mathrm{b}}$ & 14,8 & 15,3 & 7,7 & 0,0012 & 0,0014 & 0,5932 \\
\hline TIA, h & $0,29^{\mathrm{a}}$ & $0,22^{b}$ & $0,22^{\mathrm{b}}$ & $0,23^{\mathrm{ab}}$ & 0,25 & 0,23 & 78,3 & 0,0291 & 0,4527 & 0,6491 \\
\hline
\end{tabular}

a,b,c Médias com letras distintas na linha, diferem pelo teste t de student ao nível de $5 \%$.

$\mathrm{CV}=$ coeficiente de variação;

TA = Tempo de alimentação; TR = tempo de ruminação; $\mathrm{TO}=$ tempo de ócio; TIA = tempo de ingestão de água;

Fonte: Autores. 
Os períodos gastos com a ingestão de alimento são intercalados com um ou mais períodos de ruminação ou ócio. Os tempos de ruminação foram semelhantes entre as avaliações do primeiro, terceiro e quarto períodos e diferiram da avaliação do segundo período, que apresentou o menor tempo de ruminação. Já o tempo de ócio foi maior na avaliação do segundo período, porém não diferiu da avaliação do terceiro período e o menor tempo de ócio foi observado na avaliação do primeiro período. A atividade de ruminação é um processo importante para os ruminantes, pois possibilita que o bolo alimentar seja remastigado e ensalivado até atingir tamanho de partícula adequado o que, além de propiciar maior área de contato com os microrganismos ruminais, possibilita o escape para as partes posteriores do trato gastrintestinal.

Em animais confinados, o tempo destinado à ruminação representa aproximadamente oito horas por dia (Freitas et al., 2010; Missio et al., 2010). No entanto, a duração e os padrões de distribuição dos ciclos de ruminação são influenciados pelas atividades de ingestão, pela forma física da dieta, pelo teor de parede celular dos volumosos, frequências de alimentação e quantidade e qualidade de alimento consumido (Murphy et al., 1983, Van Soest, 1994; Dado \& Allen, 1995), o que ocorreu no presente trabalho, apresentando valores inferiores em função da composição e forma física da dieta. A diminuição do tempo destinado à ruminação e o aumento do tempo de descanso dos animais são importantes, pois implicam na diminuição de atividade física, que demanda energia (Missio et al., 2010).

O tempo de ingestão de água foi maior na avaliação do primeiro período em relação ao segundo e terceiro períodos, e semelhante ao quarto período. A ingestão de água é uma variável que pode ser influenciada por vários fatores, como consumo de matéria seca, características dos alimentos e condições ambientais.

Os tempos de alimentação e ingestão de água não diferiram entre as dietas com ou sem ureia encapsulada, justificados possivelmente pelo semelhante consumo de matéria seca. $\mathrm{O}$ tempo de ócio foi maior $(\mathrm{P}<0,05)$ para os animais que receberam a dieta sem ureia encapsulada em relação a dieta com, em que os animais permaneceram em ócio por 15,3 e 14,8 horas diárias, respectivamente. Já para o tempo de ruminação, os animais que receberam a dieta contendo ureia encapsulada permaneceram mais $(\mathrm{P}<0,05)$ tempo ruminando em comparação com os que receberam a dieta sem ureia encapsulada, apresentando valores de 5,4 e 5,0 horas, respectivamente. Estes resultados podem ser explicados pela proporção dos ingredientes das dietas serem diferentes, principalmente o bagaço de cana cru, pois a dieta com ureia encapsulada apresentava 7\% a mais deste ingrediente em relação à outra dieta (Tabela 1), elevando os teores de fibra detergente neutro.

Neste tipo de dieta, o bagaço de cana tem sido utilizado como fonte de fibra para estimular a ruminação e evitar problemas metabólicos para ruminantes. A ruminação pode ser influenciada pelo conteúdo nutricional da dieta, principalmente pelo teor de parede celular (Van Soest, 1994). Segundo Leite et al. (2020), as partículas longas formam um material flutuante no rúmen e proporcionam o estímulo necessário para se desencadear a atividade de ruminação, depois de vários ciclos de ruminação, as partículas fibrosas longas são reduzidas a um tamanho tal que possam escapar ao rúmen. Por isso dietas com maiores teores de fibras necessitam de maior tempo de ruminação para serem potencialmente degradadas.

Freitas et al. (2010) e Missio et al. (2010) demonstram que em geral ocorre aumento do tempo de ruminação conforme eleva-se o teor de fibra detergente neutro. Pereira et al. (2007) observaram que novilhas leiteiras alimentadas com dietas contendo $60 \%$ de fibra detergente neutro despenderam 28,$0 ; 15,8$ e 20,2\% a mais de tempo envolvidas com a atividade de mastigação, ruminação e ruminação total, respectivamente, quando comparadas com aquelas alimentadas com rações contendo $30 \%$ de fibra detergente neutro.

A eficiência de alimentação da matéria seca não apresentou interação significativa (Tabela 4). Para as dietas, a eficiência de alimentação foi semelhante. No entanto, para os períodos verificaram maiores eficiências de alimentação da matéria seca nas avaliações do terceiro e segundo períodos, seguido pelo primeiro e com menor eficiência no quarto período. Estes resultados estão relacionados, possivelmente pela variação no consumo de matéria seca e tempo de alimentação. Barros et al (2020) verificaram semelhança para a eficiência de alimentação ( $\mathrm{g}$ de matéria seca e fibra detergente neutro/min) ao 
comparar níveis de glicerol em substituição o grão de milho na dieta de ovinos, e explicaram pela similaridade do consumo de matéria seca e fibra detergente neutro.

Cirne et al. (2014), avaliaram níveis de proteína na dieta de ovinos, verificando que a eficiência de alimentação (kg matéria seca/hora) se elevou linearmente, sendo que, para cada $1 \%$ de aumento na porcentagem de proteína bruta da dieta, houve acréscimo de $0,038 \mathrm{~kg} /$ hora. E justificaram esse comportamento, em função da semelhança entre o consumo de matéria seca e elevação no tempo de ingestão, uma vez que a eficiência de alimentação foi obtida mediante a divisão do consumo de matéria seca pelo tempo total despendido em alimentação em 24 horas.

A eficiência de ruminação da matéria seca teve interação significativa. Na avaliação do primeiro período os animais que receberam a dieta sem ureia encapsulada tiveram maior eficiência de ruminação em relação aos animais que receberam a dieta com ureia encapsulada. Nas demais avaliações a eficiência de ruminação foi semelhante entre os animais que receberam as diferentes dietas. A eficiência de ruminação foi maior na avaliação do segundo período, independente da dieta. Esse resultado pode ser explicado pela variação no consumo de matéria seca (Tabela 2) e tempo de ruminação (Tabela 3). Callegaro et al. (2018), descrevem em sua pesquisa a correlação entre eficiência de ruminação da matéria seca e consumo de matéria seca $(\mathrm{r}=0,8386 ; \mathrm{P}<0,0001)$ e correlação inversa com o tempo de ruminação $(\mathrm{r}=-0,5732 ; \mathrm{P}<0,0009)$.

Tabela 4. Eficiências de alimentação da matéria seca (EAMS) e ruminação da matéria seca (ERMS) de bovinos da raça Nelore não castrados alimentados com ureia, encapsulada (CUE) ou não (SUE), conforme os períodos de alimentação em confinamento

\begin{tabular}{|c|c|c|c|c|c|c|c|c|c|}
\hline \multirow{2}{*}{ Dieta (D) } & \multicolumn{4}{|c|}{ Períodos (P) } & \multirow[b]{2}{*}{ Médias } & \multirow{2}{*}{$\begin{array}{l}\mathrm{CV} \\
(\%)\end{array}$} & \multicolumn{3}{|c|}{ Probabilidade } \\
\hline & $1^{\circ}$ & $2^{\circ}$ & $3^{\circ}$ & $4^{\circ}$ & & & $\mathrm{P}$ & $\mathrm{D}$ & $\mathrm{P} * \mathrm{D}$ \\
\hline & \multicolumn{4}{|c|}{ EAMS, kg/h de ingestão } & & \multirow{4}{*}{26,6} & \multirow{4}{*}{0,0004} & \multirow{4}{*}{0,8574} & \multirow{4}{*}{0,2131} \\
\hline SUE & 2,70 & 2,81 & 2,70 & 2,25 & 2,62 & & & & \\
\hline CUE & 2,50 & 2,80 & 3,00 & 2,23 & 2,63 & & & & \\
\hline \multirow[t]{2}{*}{ Médias } & $2,60^{\mathrm{b}}$ & $2,80^{\mathrm{a}}$ & $2,85^{\mathrm{a}}$ & $2,24^{\mathrm{c}}$ & & & & & \\
\hline & \multicolumn{4}{|c|}{ ERMS, kg/h de ruminação } & & \multirow{4}{*}{23,2} & \multirow{4}{*}{$<0,0001$} & \multirow{4}{*}{0,0557} & \multirow{4}{*}{0,0138} \\
\hline SUE & $1,84^{\mathrm{b}}$ & $2,18^{a}$ & $1,64^{\mathrm{c}}$ & $1,50^{\mathrm{c}}$ & 1,79 & & & & \\
\hline CUE & $1,50^{\mathrm{c}}$ & $2,21^{\mathrm{a}}$ & $1,59^{c}$ & $1,44^{\mathrm{c}}$ & 1,68 & & & & \\
\hline Médias & 1,67 & 2,20 & 1,62 & 1,47 & & & & & \\
\hline
\end{tabular}

a,b,c Médias com letras distintas, diferem pelo teste $\mathrm{t}$ de student ao nível de 5\%.

$\mathrm{CV}=$ coeficiente de variação;

Fonte: Autores.

\section{Conclusão}

O comportamento ingestivo é alterado ao longo dos dias em que os novilhos ficam confinados, bem como pela composição da dieta. Ademais, como possibilidades de pesquisas futuras, avaliar em outras categorias de bovinos se os dados apresentam as mesmas tendências. Além disso, a possibilidade de formular rações, com ureia encapsulada ou não, associadas a outros ingredientes. 


\section{Agradecimentos}

As empresas Nutron Alimentos LTDA, Alltech do Brasil e Grupo JBS-Friboi pelo auxílio técnico e financeiro para a realização deste projeto e à Coordenação de Aperfeiçoamento de Pessoal de Nível Superior (CAPES), pela bolsa de Doutorado concedida ao autor.

\section{Referências}

Arboitte, M. Z., Restle, J., Alves Filho, D. C., Brondani, I. L., Silva, J. H. S. da, Nörnberg, J. L. \& Kuss, F. (2004). Desempenho em confinamento de novilhos 5/8 Nelore - 3/8 Charolês abatidos em diferentes estádios de desenvolvimento. Revista Brasileira de Zootecnia, 33(4), 947-958.

Arnold, G. W. (1985) Ingestive behaviour. In: Fraser, A. F. (Ed.). Ethology of farm animals, a comprehensive study of the behavioural features of the common farm animals. New York, Elsevier, p. 183-200.

Barros, R. P., Araújo, F. L. de, Pimentel, V. A., Fortes, A. de C. \& Barros, L. J. N. M. (2020). Comportamento ingestivo de cordeiros suplementados com níveis de glicerol em substituição ao milho. Research, Society and Development, 9(9), 1-15.

Callegaro, A. M., Alves Filho, D. C., Pizzuti, L. A. D., Segabinazzi, L. R., Brondani, I. L. \& Martini, A. P. M. (2018) Comportamento ingestivo de novilhos mestiços terminados em confinamento alimentados com borra de soja. Ciência Animal Brasileira, 19(e-28150), 1-12.

Cirne, L. G. A., Oliveira, G. J. C., Jaeger, S. M. P. L., Bagaldo, A. R., Leite, M. C. P., Rocha, N. B., Macedo Junior, C. M. \& Oliveira, P. A. (2014) Comportamento ingestivo de cordeiros em confinamento, alimentados com dieta exclusiva de concentrado com diferentes porcentagens de proteína. Arquivo Brasileiro de Medicina Veterinária e Zootecnia, 66(1), 229-234.

Cruz, G. M. da, Esteves, S. N., Tullio, R. R., Alencar, M. M. de \& Oliveira, M. C. de S. (2004). Peso de abate de machos não castrados para produção do bovino jovem. 1. Desempenho em confinamento e custos de produção. Revista Brasileira de Zootecnia, 33(3), 635-645.

Dado, R. G. \& Allen, M. S. (1995) Intake limitations, feeding behavior, and rumen function of cows challenged with rumen fill from dietary fiber or inert bulk. Journal of Dairy Science, 78(1), 118-133.

Delavaud, C., Bocquier, F., Chilliard, Y., Keisler, D. H., Gertler, A. \& Kann, G. (2000). Plasma leptin determination in rimunants: effect of nutritional status and body fatness on plasma leptin concentration assessed by a specific RIA in sheep. Journal of Endocrinology. 165(2), 519-526.

Ferreira, R. N., Oliveira, E. R., Orsine, G. F., Paula, A. A., Oliveira, L. G., Bittencourt, A. R. \& Souza, S. N. (2005). Liberação de nitrogênio amoniacal no rúmen com o uso de uréia encapsulada com polímero (Optigen 1200 Alltec). In: Reunião Anual da Sociedade Brasileira de Zootecnia, 42, 2005, Goiânia. Anais... Goiânia, 2005. CD-ROOM.

Freitas, L. S., Silva, J. H. S., Segabinazzi, L. R., Silva, V. S. da, Alves Filho, D. C. \& Brondani, I. L. (2010) Substituição de silagem de milho por silagem de girassol na dieta de novilhos em confinamento: comportamento ingestivo. Revista brasileira de Zootecnia, 39(1), $225-232$.

Guimarães, Y. L. F., Debortoli, E. de C., Santos, J. dos \& Gopinger, E. (2020). Comportamento ingestivo de bovinos em diferentes sistemas de produção uma revisão sistemática de estudos científicos. Research, Society and Development, 9(10), 1-14.

Jorge, A. M., Fontes, C. A. A., Freitas, J. A., Rodriguez, L. R. R. \& Resende, F. D. (1997). Ganho de peso e de carcaça, consumo e conversão alimentar de bovinos e bubalinos, abatidos em dois estágios de maturidade. Revista Brasileira de Zootecnia, 26(4), 806-812.

Leite, H. M. de S., Batista, N. V., Lima, A. F. de, Silva, L. A. da, Oliveira, J. T. M. C. B. de, Firmino, S. S., Silva, M. R. L. \& Lima, P. de O. (2020). Desempenho e comportamento ingestivo de cordeiros alimentados com dieta de alto grão. Research, Society and Development, 9(10), 1-21.

Mello, R. O., Queiroz, A. C., Resende, F. D., Faria, M. H. de, Henrique, D. S. \& Maldonado, F. (2010). Bionutritional efficiency of crossbred beef cattle finished on feedlot and slaughtered at different body weights. Revista Brasileira de Zootecnia, 39(3), 582-593.

Mertens, D. R. (1994) Regulation of forage intake. In: Fahey Jr., G. C. (Ed) Forage quality, evaluation and utilization. Madison: American Society of Agronomy, 1, 450-493.

Millen, D. D., Pacheco, R. D. L., Arrigoni, M. D. B., Galyean, M. L. \& Vasconcelos, J. T. (2009). A snapshot of management practices and nutritional recommendations used by feedlot nutritionists in Brazil. Journal of Animal Science 87(10), 3427-3439.

Missio, R. L., Brondani, I. L., Alves Filho, D. C., Silveira, M. F. da, Freitas, L. da S. \& Restle, J. (2010) Comportamento ingestivo de tourinhos terminados em confinamento, alimentados com diferentes níveis de concentrado na dieta. Revista Brasileira de Zootecnia, 39(7), 1571-1578.

Murphy, M. R., Baldwin, R. L, Ulyatt, M. J. \& Koong, L. J. (1983) A quantitative analysis of rumination patterns. Journal of Animal Science, 56(5), 12361240 .

NRC - National Research Council. (2016) Nutrient requirements of beef cattle. (8a ed.), revised edition. National Academy Press, Washington, D.C.

Oliveira, C. A. \& Millen, D. D. (2014). Survey of the nutritional recommendations and management practices adopted by feedlot cattle nutritionists in Brazil. Animal Feed Science and Technology, 197, 64-75.

Paulino, P. V. R., Carvalho, J. C. F., Cervieri, R. C., Terêncio, P. \& Vargas, A. (2010) Estratégias de adaptação de bovinos de corte às rações com teores elevados de concentrado. In: IV CLANA - IV Congresso Latino-Americano de Nutrição Animal. Anais... 351-362. 
Research, Society and Development, v. 10, n. 6, e41110615890, 2021

(CC BY 4.0) | ISSN 2525-3409 | DOI: http://dx.doi.org/10.33448/rsd-v10i6.15890

Pereira, J. C., Cunha, D. N. F. V., Cecon, P. R. \& Faria, E. de S. (2007) Comportamento ingestivo e taxa de passagem de partículas em novilhas leiteiras de diferentes grupos genéticos submetidas a dietas com diferentes níveis de fibra. Revista Brasileira de Zootecnia, 36, 2134-2142.

Pinto, A. C. J. \& Millen, D. D. (2018). Nutritional recommendations and management practices adopted by feedlot cattle nutritionists: the 2016 brazilian survey. Canadian Journal of Animal Science, 99, 392-407.

Santos, J. F, Dias Júnior, G. S., Bitencourt, L. L, Lopes, N. M., Siécola Júnior, S., Silva, J. R. M., Pereira, R. A. N. \& Pereira, M. N. (2011) Resposta de vacas leiteiras à substituição parcial de farelo de soja por ureia encapsulada. Arquivo Brasileira de Medicina Veterinária e Zootecnia, 63(2), 423-432.

SAS Institute. (2001) Statistical analysis system user's guide. Version 8.02. Cary: Statistical Analysis System Institute.

Silva, D. J. \& Queiroz, A. C. (2002) Análise de alimentos: métodos químicos e biológicos. (3a ed.), Universidade Federal de Viçosa, 235p.

Silva, J. F. C. (2011). Mecanismos reguladores de consumo. In: Berchielli, T. T., Pires, A. V. \& Oliveira, S. G. Nutrição de ruminantes. Funep, 3 , $61-82$.

Van Soest, P. J. (1994) Nutritional ecology of the ruminant. (2a ed.), Cornell University Press., 476p. 